\title{
Evanescent wave DNA-aptamer biosensor based on long period gratings for the specific recognition of $E$. coli outer membrane proteins
}

\author{
R.B. Queirós ${ }^{\text {a,* }}$, C. Gouveia ${ }^{a}$, J.R.A. Fernandes ${ }^{a, b}$, P.A.S. Jorge ${ }^{a}$ \\ a INESC TEC (Coordinated by INESC Porto), Rua do Campo Alegre, 687, 4150-179 Porto, Portugal \\ ${ }^{\mathrm{b}}$ Universidade de Trás-os-Montes e Alto Douro, Apartado 1013, 5001-801 Vila Real, Portugal
}

\section{A R T I C L E I N F O}

\section{Article history:}

Received 25 March 2014

Received in revised form

9 June 2014

Accepted 12 June 2014

Available online 3 July 2014

Keywords:

Aptamer

Biosensor

E. coli

Label-free

LPGs

\begin{abstract}
A B S T R A C T
An evanescent wave fiber optic sensor for detection of Escherichia coli (E. coli) outer membranes proteins (EcOMPs) using long period gratings (LPGs) as a refractometric platform is presented. The sensing probes were attained by the functionalization of LPGs inscribed in single mode fiber using two different methods of immobilization; electrostatic assembly and covalent binding. The resulting label-free configuration enabled the specific recognition of EcOMPs in water by monitoring the resonance wavelength shift due to refractive index changes induced by binding events. The sensors displayed linear responses in the range of $0.1 \mathrm{nM}$ to $10 \mathrm{nM}$ EcOMPs with sensitivities of $-0.1563 \pm 0.005 \mathrm{~nm}$ decade $^{-1}$ [EcOMP, M] (electrostatic method) and $-0.1597 \pm 0.004 \mathrm{~nm}^{\text {decade }}{ }^{-1}$ [EcOMP, M] (covalent method). The devices could be regenerated (under low $\mathrm{pH}$ conditions) with a deviation less than $0.1 \%$ for at least three subsequent detection events. The sensors were also applied to spiked environmental water samples.
\end{abstract}

(c) 2014 Elsevier B.V. All rights reserved.

\section{Introduction}

The increasing need for devices capable of performing reliable, fast and in situ measurements in the field of biochemical detection is guiding researchers to look for new technologies (Baldini et al., 2012). One possibility is provided by optical refractometers, which measure the change in refractive index associated with a biochemical response. In these type of sensors the crucial phenomenon is interaction, typically evanescent, of an optical signal with a sensitive layer which experiences a change on its own refractive index in the presence of an analyte. This variation will cause a change in light propagation conditions leading to a change in the properties of the transmitted optical wave (intensity, frequency, phase). In the specific case of biological sensors the binding events cause an increase of the mass on the surface which results in an increase of the effective refractive index of the propagated optical wave. Fiber optic refractometers are increasingly popular due to the benefits that optical fiber technology offers such as: immunity to electromagnetic interferences, chemical and biological inertness, small size, and capability for in-situ, real-time, remote, and distributed sensing. Among the most popular fiber optic refractometric devices for biosensing application are surface plasmon resonance (Slavík et al., 2001), fiber tapers (Zibaii et al., 2010),

\footnotetext{
* Corresponding author. Tel.: +351 220402 301; fax: +351220 402437.

E-mail addresses: raquelbqueiros@gmail.com, rqueiros@inescporto.pt (R.B. Queirós).
}

etched fiber Bragg gratings (Chryssis et al., 2005) and LPGs (DeLisa et al., 2000). Among these, LPGs is the only one where the physical integrity of the fiber is preserved thus increasing the robustness of the sensor to use and handling (Pilla et al., 2012).

A LPG consists of a periodic modulation of the fiber core refractive index with a period $(\Lambda)$ in the range of hundreds of microns. This perturbation must comply with the phase matching condition between the core fundamental mode and a forward propagating cladding mode for the optical fiber. Thereby, in an LPG, the core mode can be coupled into one or more of the several cladding modes of the fiber whenever the matching condition is verified, resulting in several resonances centered at discrete wavelengths in the transmitted spectrum (Bhatia and Vengsarkar, 1996). This mechanism provides the possibility of interaction between the optical signal and the external medium through the evanescent field of the cladding modes and also means that these devices are intrinsically sensitive to the surrounding refractive index (SRI) (Patrick et al., 1998). LPGs are among the most promising fiber optic-based RI transducers to be employed for unlabeled biochemical assays (Fan et al., 2008). LPGs present high sensitivity to refractive index measurement, which can be increased and tuned by properly choosing the fabrication parameters (Shu et al. 2002) and/or using high refractive index overlays (Pilla et al., 2012). In terms of fabrication, they are easier and less expensive to obtain when compared with surface plasmon resonance-based fiber optic sensors which, in turn, are usually more sensitive. Nevertheless, although this is a promising 
platform, several aspects regarding the practical implementation of biosensors using LPGs require further investigation. In this work, practical aspects of biosensors implementation using LPGs are investigated in a bacterial detection application. Namely, the influence of the type of functionalization strategy, calibration, quantification and specific detection are addressed. In particular, the present work describes the use of an Escherichia coli DNA aptamer (herein called ECA) for the determination of E. coli in water by the identification of EcOMPs using a LPG as a refractometric platform. The aptamer raised against EcOMPs (Bruno et al., 2010) containing 36 nucleotides was immobilized using two different methods of immobilization: electrostatic assembly (using a cationic polymer) and with covalent binding (using an organofunctional alkoxysilane molecule). The functionalization of the bare LPG by these two different methods of immobilization originate sensing heads with different characteristics that were characterized and tested against EcOMPs and validated with spiked environmental water samples.

\section{Materials and methods}

\subsection{Apparatus}

The characterization setup was constituted by a FS2200SA Braggmeter from Fibersensing, SA (Portugal), a fluidic system containing 2 reaction chambers with capacity of $750 \mu \mathrm{L}$, that operate in continuous mode by manual injection and a laptop with LabVIEW software that receives and processes the readout information. Each sensing element was characterized using the FS2200SA Braggmeter (working in the 1500-1600 nm range with $2.5 \mathrm{pm}$ of resolution) and modified to measure signals both in reflection and transmission mode in separate channels. A schematic illustration of the setup is presented in Fig. 1. The inset of the figure shows a scheme of the LPG structure with the description of the mode coupling mechanism and the spectra of incoming light and of transmitted light, it also illustrates the shift of the resonance peak for increasing surrounding refractive index (SRI) of the wavelength field. LPGs are intrinsically sensitive to external refractive index, exhibiting changes in the position of the resonance wavelength $\left(\lambda_{R}\right)$. The resonant coupling of light to a particular cladding mode is given by the phase matching condition (Erdogan, 1997), Eq. (1).

$\lambda_{R}^{m}=\left(n_{\text {eff,core }}-n_{\text {eff,cladding }}^{m}\right) \Lambda$

where $\Lambda$ is the grating period, and $n_{\text {eff,core }}$ and $n_{\text {eff,cladding }}$ are the effective indexes of the guided modes and $m$ th-cladding mode, respectively. Following the phase matching condition, a change in the surrounding refractive index (SRI) will induce a shift in the resonance wavelength due to the variation of the $n_{\text {eff,cladding, }}^{m}$ which is dependent on the external refractive index.

The changes in the LPG resonant peak are monitored in real time by the unit. The real time tracking of the spectral peak position was implemented using a standard threshold peak detection algorithm from Labview software. In addition, at each stage during the sensing head assembly and the affinity assay the spectrum could also be recorded. The fibers were properly fixed with fiber clamps in both sides, and a small mechanical tension was applied with a translation stage to avoid strain/curvature cross sensitivity. The center wavelength of each resonance peak is given by the phase matching condition, which is expressed as a function of the difference of the effective index of the core and $m$ th cladding mode and the grating period. Thus, any external parameter able to modify those will modify the phase matching condition and consequently the LPG center wavelength. In such way the LPG is sensitive to surrounding environment conditions such as temperature, strain, curvature and refractive index (Vengsarkar et al., 1996 and Bhatia et al. 1996). The experiments were carried out in a temperature-controlled environment with a precision of $\pm 0.1^{\circ} \mathrm{C}$. Furthermore, the test chamber allowed to submit the LPG to a fixed pre-tension to eliminate cross-sensitivity to strain and curvature.

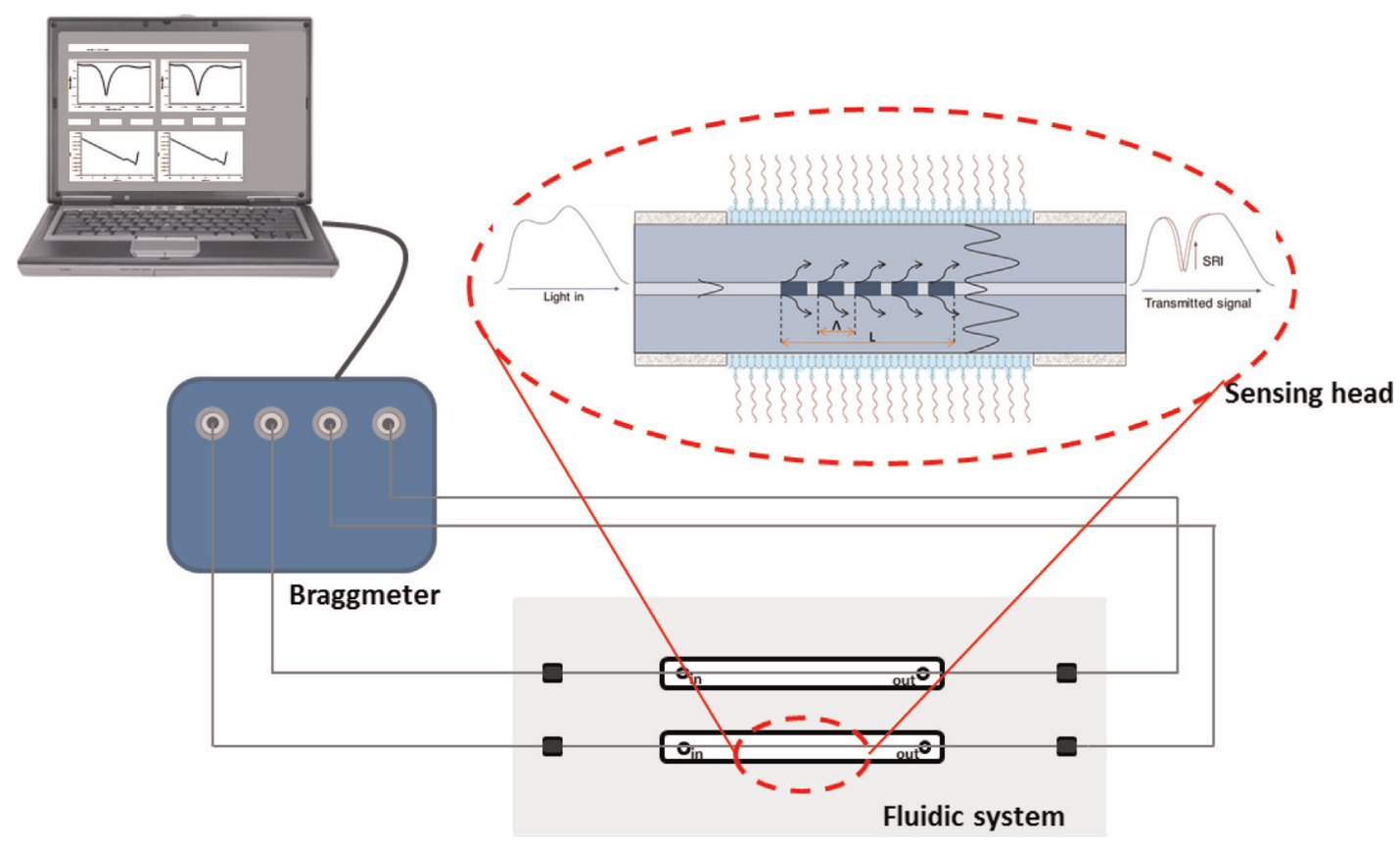

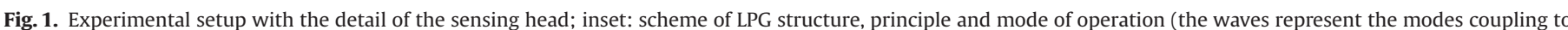

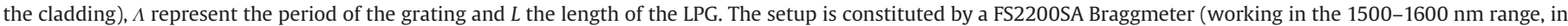

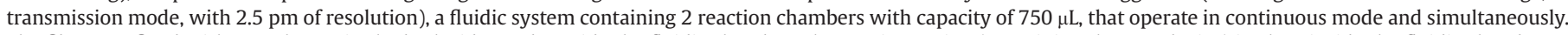

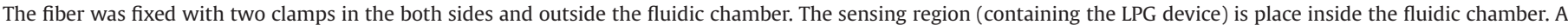
laptop with LabVIEW software that receives and processes the readout information is also part of the setup. 


\subsection{Reagents and solutions}

A lawn of E. coli ATCC $^{\mathrm{R}}$ 8739 ${ }^{\mathrm{TM}}$ was purchased from ATCC (USA) and EcOMPs were extracted according to Bruno et al., (2010). The E. coli ATCC $^{\mathbb{R}} 8739^{\mathrm{TM}}$ is a fecal strain non-pathogenic. Nonetheless, the targeted proteins are expressed in both non-pathogenic and pathogenic $E$. coli, so the pathogenic nature of the strain used to obtain the analyte does not involve a change in the analytical characteristic of the assay. "36 mer DNA oligo (5'-amine-GTC TGC GAG CGG GGC GCG GGC CCG GCG GGG GAT GCG-3') synthesis scale: $0.2 \mu \mathrm{M}$, purification HPLC, $5^{\prime}$ modification-amine was purchased from Sigma-Aldrich (Spain). Salts for buffer solutions were prepared in double-deionized water, $<2 \mathrm{M} \Omega \mathrm{cm}$ at $25^{\circ} \mathrm{C}$ from ELGASTAT-UHQ (UK). Hydrochloric acid $(\mathrm{HCl})$ was purchased from Merck (Germany), magnesium chloride was purchased from Panreac (Spain), (Tris(hydroxymethyl)aminomethane (Tris) were obtained from Fluka (Germany), (3-aminopropyl)triethoxysilane 97\% (APTES) was obtained from ABCR (Germany), 0.1\% (W/V) polyL-lysine (PLL), dimethyl suberimidate (DMSI), potassium chloride $(\mathrm{KCl})$, sodium chloride $(\mathrm{NaCl})$, SSPE buffer $20 \mathrm{x}$ concentrate $(1 \times=0.150 \mathrm{M}$ sodium chloride, $0.010 \mathrm{M}$ sodium phosphate, $0.001 \mathrm{M}$ EDTA), Tris-EDTA, microcystin-LR (Mcyst-LR) were obtained from Sigma (Germany). The buffers used in this work were: resuspension buffer: TE $(10 \mathrm{mM}$ Tris $\mathrm{pH} 7.5-8.0 ; 1 \mathrm{mM}$ EDTA); immobilization buffer: SSPE (1:10), (diluted from $20 \times$ SSPE); affinity buffer $(50 \mathrm{mM}$ Tris/ $\mathrm{HCl} \mathrm{pH} 7.4,250 \mathrm{mM} \mathrm{NaCl}$, $5 \mathrm{mM} \mathrm{MgCl}_{2}$ ) and measurement buffer (10 mM Tris/HCl pH 7.4, $100 \mathrm{mM} \mathrm{KCl}$ ). All the reagents were of analytical grade.

\subsection{Experimental methods}

\subsubsection{Extraction and determination of EcOMPs concentration}

A lawn of $E$. coli ATCC $^{\circledR} 8739^{\mathrm{TM}}$ was grown on a blood agar plate overnight at $37^{\circ} \mathrm{C}$. Bacteria were washed from the plate with the extraction buffer and transferred to $10 \mathrm{ml}$ of cold $1.5 \mathrm{M} \mathrm{MgCl}_{2}$ in sterile nuclease-free water. The suspension of bacteria was left overnight at $4{ }^{\circ} \mathrm{C}$ to allow the chaotropic action of $\mathrm{MgCl}_{2}$ to extract the membrane proteins. The resultant pellet was washed again with buffer and centrifuged (Bruno et al. 2010). The liquid was collected and the final concentration of EcOMPs was estimated spectrophotometrically at $280 \mathrm{~nm}$ (Jing and Bowser, 2011).

\subsubsection{LPGs fabrication and characterization}

The gratings were fabricated in-house by using an electric-arc setup (Rego et al. 2001). The LPGs were written in a standard SMF28 fiber (Corning, USA) using the following set of fabrication parameters: an axial tension of $50.0 \mathrm{mN}$, arc discharges with an electric current of $9 \mathrm{~mA}$ and arc duration of $0.5 \mathrm{~s}$.

The fiber is illuminated with a white light source and the spectrum is monitored in real time by using an optical spectrum analyzer (OSA), Ando AQ-6315B, with a maximum wavelength resolution of $0.5 \mathrm{~nm}$. The sequence arc discharge/fiber displacement is repeated several times until a required attenuation value for the resonance peak (monitored with the OSA) is obtained. As the discharges are being made the resonance peak increases in depth and becomes narrower until a maximum loss is reached. The grating period was $396 \mathrm{~mm}$ in order to produce a resonant wavelength at approximately $1560 \mathrm{~nm}$, corresponding to the 6th order cladding mode. The period of the grating was chosen according with previous work (Caldas et al., 2009). The length of the used LPGs are around $40 \mathrm{~mm}$ depending on the number of periods necessary to achieve the desired resonant wavelength and loss (between 80-90 periods). This way, deriving from this fabrication process, the particular LPGs may differ in their spectral shape and precise value of attenuation. Nevertheless their sensitivity to refractive index depends specifically on the cladding order mode excited and is identical for all LPG fabricated. From this point forward, whenever LPG resonance will be referred, it will be relatively to the 6 th order cladding mode.

The sensitivity of the LPG to the SRI was also studied for the range between 1.321-1.345 (Fig. S1), corresponding to the range of interest for biological measurements (Zibaii et al., 2010), and was found to be about $147 \pm 3 \mathrm{~nm} \mathrm{RIU}{ }^{-1}$. The LPG was characterized in terms of SRI and its transmission spectra for different SRIs were recorded. The SRI was changed by using solutions of distilled water with different percentages of ethylene glycol at constant temperature $\left(25^{\circ} \mathrm{C}\right)$. Each liquid sample had its RI previously characterized with an Abbe refractometer using the sodium D line $(589 \mathrm{~nm})$. The necessary adjustments, considering the sensing head operation at $1550 \mathrm{~nm}$, can be made using the Cauchy equation with the respective coefficients (Fogg et al., 1955; ElKashef, 2000). The corrected sensitivity of the device considering the wavelength of the studied resonance is around $150 \mathrm{~nm} \mathrm{RIU}{ }^{-1}$ (Gouveia, 2013). The LPGs have also a known sensitivity to temperature variations and a characterization was performed to determine if their thermal behavior was within the expected values. By monitoring its behavior while immersed in cooling hot water, the thermal sensitivity of the sensing device was obtained. From the slope of the calibration curve presented in Fig. S2 it can be estimated a sensitivity of $113 \pm 1 \mathrm{pm}^{\circ} \mathrm{C}^{-1}$. Therefore, during the experiments the thermal fluctuations was kept below $\pm 0.1^{\circ} \mathrm{C}$, thus avoiding cross-sensitivity to this parameter.

\subsubsection{Sensing heads assembly}

The LPGs surface were functionalized using two different processes; an electrostatic method, using PLL as cationic polymer and a covalent method, using APTES. Prior to functionalization of the LPG, they were cleaned by immersion in $5 \mathrm{M} \mathrm{HCl}$ for $30 \mathrm{~min}$ at room temperature, followed by rinsing in DD water and with Tris$\mathrm{HCl}$ buffer. The sensing head of type $1 \mathrm{SH} 1$ were coated with $0.1 \%$ PLL for $30 \mathrm{~min}$ at room temperature and then were washed with Tris- $\mathrm{HCl}$ buffer. The silanization of the LPG's surface, for the assembly of sensing head of type 2 ( $\mathrm{SH} 2$ ), was performed by immersing the cleaned grating sample in fresh 10\% APTES (in ethanol) for $1 \mathrm{~h}$, also at room temperature. For activation of the fiber surface, the silanized LPGs were immersed in $25 \mathrm{mM}$ DMSI in Tris- $\mathrm{HCl}$ buffer solution for $30 \mathrm{~min}$ at room temperature. Both sensing heads were incubated with $200 \mathrm{nM}$ of ECA overnight at $4^{\circ}$ C. The sensing heads were rinsed between all steps with Tris- $\mathrm{HCl}$ buffer.

\subsubsection{EcOMPs detection assay}

The sensing heads were incubated with several varying concentrations of EcOMPs, in the affinity buffer for $1 \mathrm{~h}$. The sensor response was tested in the concentration range from $0.1 \mathrm{nM}$ to $30 \mathrm{nM}$. Between all concentrations steps the sensing heads were washed with Tris- $\mathrm{HCl}$ buffer and then the wavelength shift of the resonance peak was acquired with the FS2200SA and recorded with the laptop with the fiber immersed in the measurement buffer. After a complete assay the sensing heads were washed with a $2 \mathrm{M} \mathrm{HCl}$ solution for $30 \mathrm{~min}$, to regenerate the DNA probe for reuse.

\section{Results and discussion}

\subsection{Sensing heads characterization - SEM and AFM analysis}

SEM and AFM analysis were conducted over both sensing heads at the different steps of the functionalization and the assembling process. The obtained images of SEM and AFM are shown in 


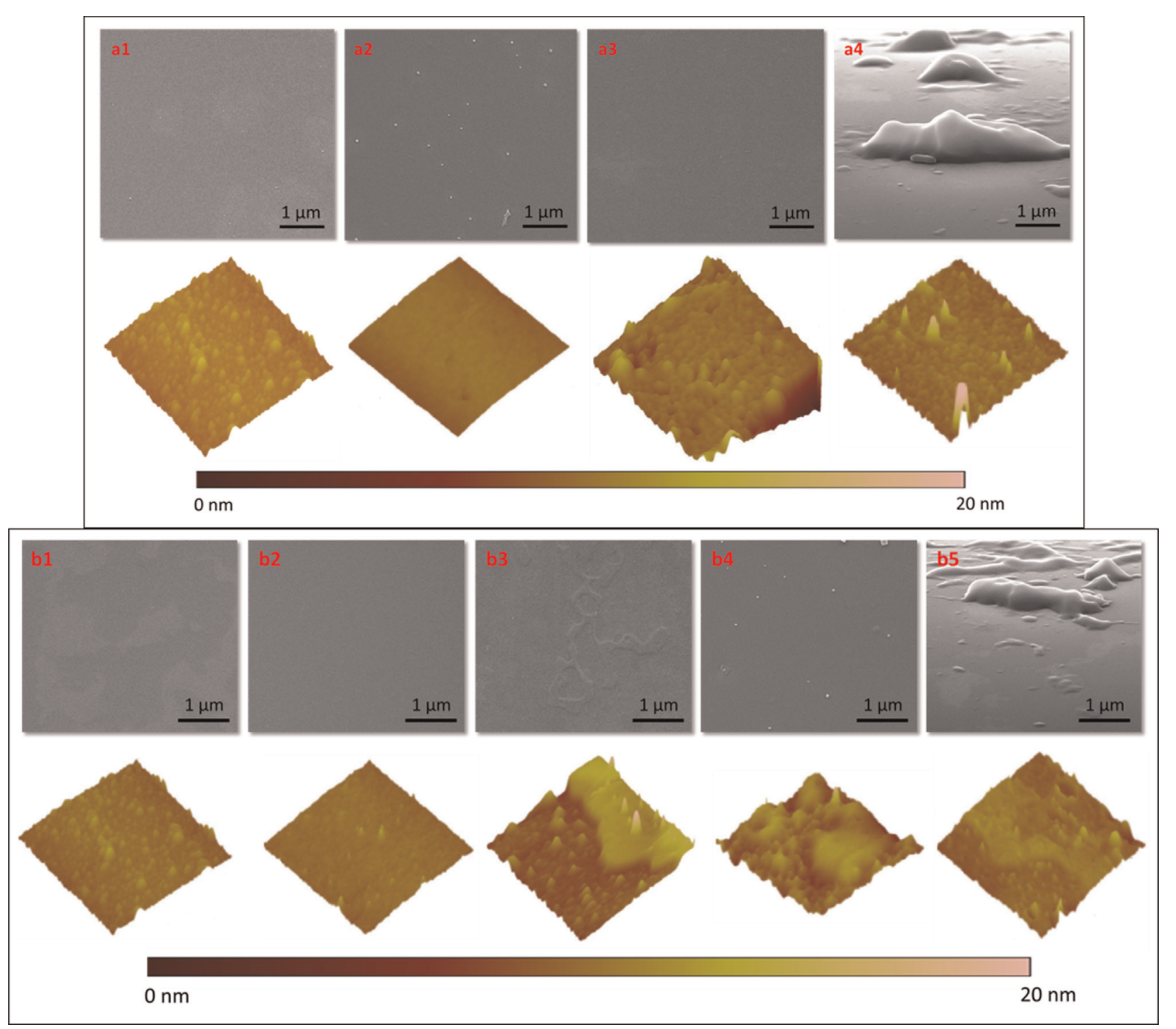

Fig. 2. SEM (top) and 3D AFM (bottom) images of SH1 for a) and SH2 for b). a1) after wash with $\mathrm{HCl}$; a2) functionalization with PLL; a3) immobilization of $1 \mu \mathrm{M}$ of ECA; a4) immobilization of $1.19 \mu \mathrm{M}$ of EcOMPs; b1) after wash with $\mathrm{HCl}$; b2) functionalization with $10 \%$ APTES; b3) functionalization with $25 \mathrm{mM}$ DMSI; b4) immobilization of $1 \mu \mathrm{M}$ of ECA; b5) immobilization of $1.19 \mu \mathrm{M}$ of EcOMPs.

Fig. 2a) and b), for sensing heads $\mathrm{SH} 1$ and $\mathrm{SH} 2$ respectively. In Fig. 2a1) and b1), a roughened surface is observed resulting from the washing with $\mathrm{HCl}$, for the activation of the surface. After the functionalization with PLL SH1 surface became smoother as can be seen in a2). The effect of the functionalization of $\mathrm{SH} 2$ in the surface of the LPGs is presented in b2) and b3), corresponding to APTES and DMSI immobilization, respectively. It was observed that the surface is smoothed by application of the aminosilane but does not appear as smooth as the surface functionalized with PLL. Also, it can be seen that the immobilization of the DMSI is not uniform, and that the layer is quite thick, about $10 \mathrm{~nm}$. After the immobilization of the ECA, a3) for SH1 and b4) for SH2, it can be observed that the AFM images of each type of sensing heads surface are quite different. The surface of $\mathrm{SH} 2$ is more heterogeneous than the SH1. A possible explanation may be that the previous layer of DMSI, was not evenly distributed, causing a heterogeneous layer of ECA. Regarding the immobilization of EcOMPs, in both sensing heads was observed that some of the bacterial mass is still present. These residuals can eventually cause perturbations on the assay due to the comparatively large mass and proximity to the surface.
Subsequent trials with EcOMPs solutions were filtered using $0.2 \mu \mathrm{m}$ filter.

\subsection{ECA LPG biosensor behavior}

The detailed principle of ECA LPG biosensor is illustrated in Fig. 1, using SH1 as an example. The LPG sensing principle primarily relies on measuring the shift in $\lambda_{R}$, due to the changes in the refractive index of fiber surroundings. The functionalized aptamer with an amine group at its $5^{\prime}$ end was bound to the optical fiber with an inscribed LPG $(\Lambda=398 \mu \mathrm{m})$ that was modified using two different processes, physisorption and covalent binding. As described in the sensing head assembly procedure, $\mathrm{SH} 1$ was modified with a cationic polymer-PLL (electrostatic interaction) and $\mathrm{SH} 2$ was modified by silanization using APTES and DMSI (silane chemistry). The polycationic nature of the PLL leads to its electrostatic attraction onto a negatively charged surface in an aqueous environment at neutral $\mathrm{pH}$.

On the other hand, to immobilize biomolecules covalently to the silica surface, as in $\mathrm{SH} 2$, a chemical bond has to be formed between a functional group of biomolecules and the amino group 

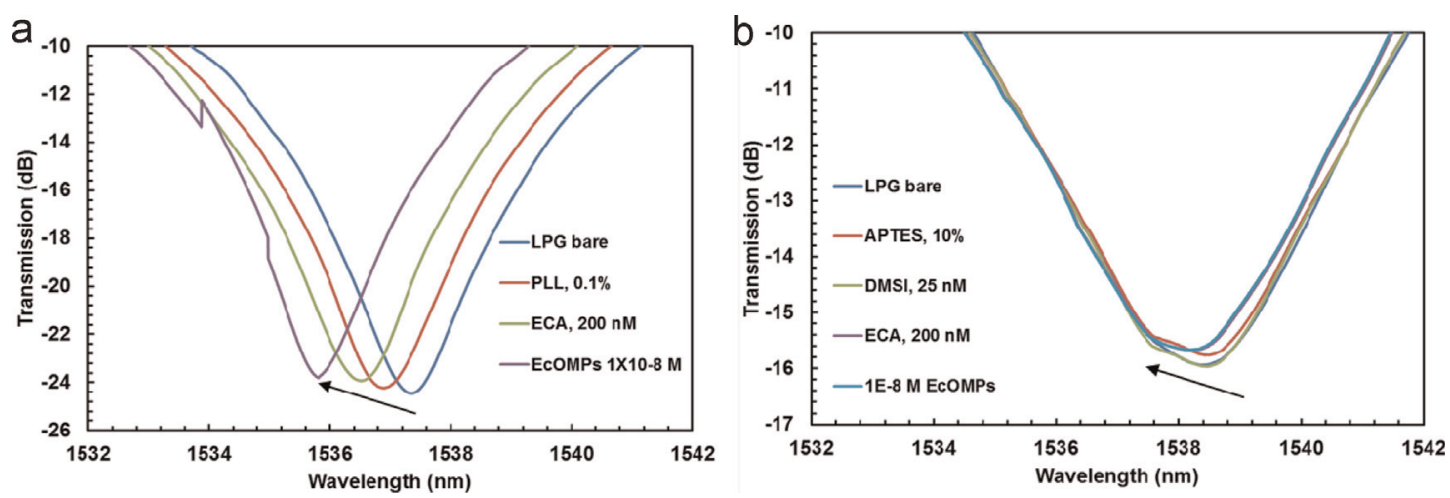

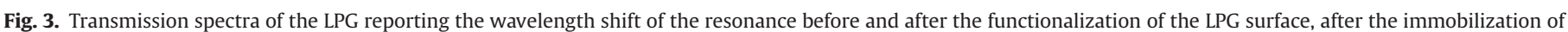
the capture probe and following affinity-assay (ECA-EcOMPs) for a) SH1 and b) SH2.

of the linker. Herein was used DMSI, a water soluble and membrane permeable cross-linking agent to convert the amino groups into reactive imidoester (Hermanson, 1996). The imidoester functional group is a specific acylating group accessible for the modification of primary amines and has minimal cross reactivity to other nucleophilic groups in proteins (Mattson et al., 1993). Both, activated sensing heads were then incubated with $200 \mathrm{nM}$ of ECA.

Subsequently, the sensing heads were incubated with several varying concentrations of EcOMPs. Between all these steps the shift of the resonance wavelength position was measured. In the presence of EcOMPs, the ECA folds around the proteins and forms an EcOMPs-ECA complex. The formation of this complex causes an increase in the effective refractive index of the cladding mode and consequently a decrease of the wavelength of the resonance. In Fig. 3 are presented the transmission spectra of the LPGs reporting the wavelength shift of the resonance during the immobilization procedure and following the affinity assay for SH1 and SH2. These results represent the medium values of 5 experiences with different LPG devices.

The wavelength shift observed is of about $1.6 \pm 0.4 \mathrm{~nm}$ from the bare LPG to the affinity assay with EcOMPs for SH1, while for SH2 the difference is of about $0.48 \pm 0.3 \mathrm{~nm}$, for $n=5$.

After the immobilization of the DMSI it was observed a wavelength shift to higher values of wavelength reducing the overall shift of the complete assay for SH2. Usually, adding layers to the LPG surface results in increasing refractive index and shift of the resonance wavelength to lower wavelengths. In this case, the opposite behavior took place. This behavior could be linked to a non-uniform deposition of the DMSI layer, as can be seen in Fig. 2b3), causing a consecutive heterogeneous layer of ECA and leading to a lower reproducibility in consecutive experiments.

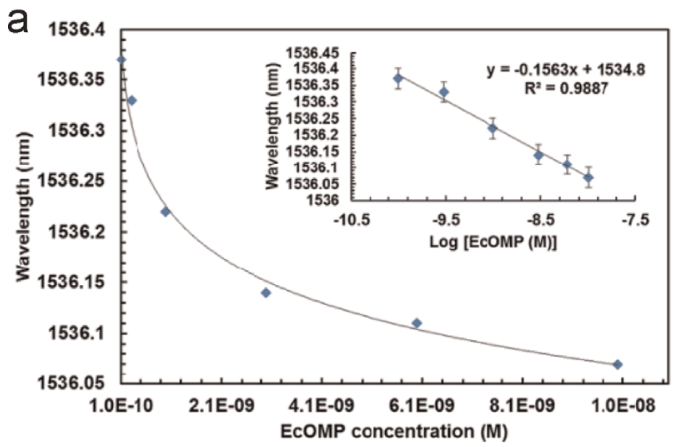

\subsection{Sensor performance against EcOMPs}

The self-assembly of the ECA and consecutively EcOMPs, onto the bare LPG surface, increases the effective index of the LPG cladding mode resulting in a shift of the resonance as a function of the surface mass (which is also increasing). The sensor performance was tested by the evaluation of $\mathrm{SH} 1$ and $\mathrm{SH} 2$ against increasing concentrations of EcOMPs ranging from $0.1 \mathrm{nM}$ to $30 \mathrm{nM}$. Measurements were conducted at room temperature in the measurement buffer.

It was found that the interaction between ECA and the EcOMPs were much faster during the first periods of the calibration and decrease along the time with the increase of the EcOMPs concentration. This behavior can mean that the surface of the sensing heads becomes rapidly covered and the linkage of arriving EcOMPs to the surface is then increasingly difficult.

In Fig. 4 is presented the LPG calibration plots relative to the wavelength shift of the resonance during the affinity assay of the complex ECA-EcOMPs ranging from $0.1 \mathrm{nM}$ to $10 \mathrm{nM}$ of EcOMPs for $\mathrm{SH} 1$ and $\mathrm{SH} 2$.

The incubation of ECA with EcOMPs for concentrations between $0.1 \mathrm{nM}$ and $10 \mathrm{nM}$ in affinity buffer progressively increases the SRI of the LPG surface and subsequently shifts the resonance for shorter wavelengths. For both sensing heads, a logarithmic relationship was verified between the resonance wavelength and the EcOMPs concentration with sensitivities of $-0.1563 \pm 0.005 \mathrm{~nm} \mathrm{decade}^{-1} \quad$ [EcOMP, M] (SH1) and $-0.1597 \pm 0.004 \mathrm{~nm}$ decade $^{-1}$ [EcOMP, M] (SH2).

Although the probe is sensitive to temperature, the effects of thermal fluctuations $\left( \pm 0.1^{\circ} \mathrm{C}\right)$ during the measurements can be neglected, since $0.1^{\circ} \mathrm{C}$ is equivalent to $11.3 \mathrm{pm}$ variation shift. However, it is a further work objective to implement a system that allows temperature independent measurements whereas LPG are

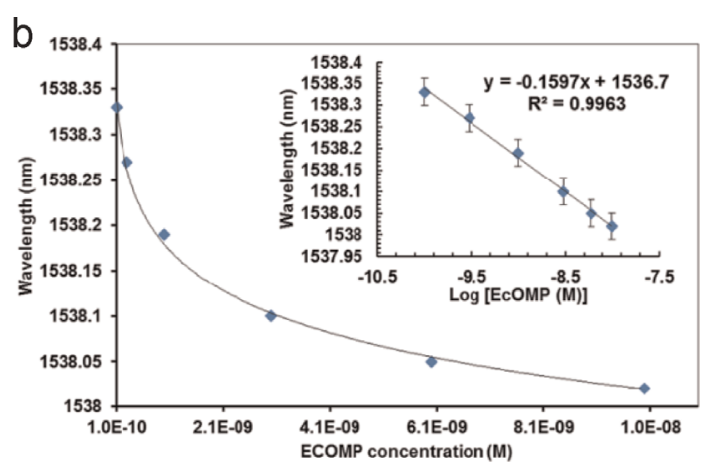

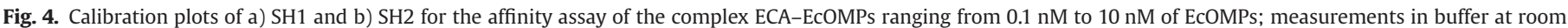
temperature; inset: linearization of calibration plots. 
highly sensitive to temperature variations. Also, some other aspects need to be taken in consideration. The reproducibility of the LPGs by electric arc is a critical issue. It is quite difficult to write gratings with the exactly same characteristics such as, length, resonance wavelength and attenuation (depth). The resonance sharpness affects the resolution (White and Fan, 2008) as well as the wavelength, because the evanescent field is proportional to the wavelength, and the length of the device is, by itself, important due to the exposed sensing area.

Moreover, the functionalization process also features some concerns. Small uniformities during functionalization process can cause surface irregularities which may cause irreproducible results. As can be seen in Fig. 2, during the SEM and AFM analysis were observed some defects during some steps of the functionalization, more pronounced in the covalent process, as can be seen in Fig. 2b3) during the functionalization with $25 \mathrm{mM}$ DMSI, which seem to result in less steady functionalized surfaces and consequently to a deformation of the resonance peak, whose behavior is noticeable in Fig. 3b).

Considering all these issues, the results obtained, corresponding to the average of a 5 assays experiment, are quite encouraging showing a reproducibility that would be adequate for preliminary alarm system of the presence of $E$. coli proteins.

In addition, all the above issues indicate that there is much room for improvement. In this context, to the best of our knowledge no information about reproducibility in biosensing with LPGs was reported so far, being therefore an open issue worth of investigation.

\subsection{Regeneration of sensing heads}

In the previous work, Queirós et al., 2013, a extensive study about regeneration was done, using other solutions such as urea or glycine, and the best results were for $\mathrm{HCl} 2 \mathrm{M}$, being the regeneration solution used in this work. The regeneration of the sensing phase was tested by carrying out consecutive binding and washing steps. The shift of the resonance was measured after the immobilization of $200 \mathrm{nM}$ of ECA (DNA probe) and after the binding between the ECA with $10 \mathrm{nM}$ EcOMPs; then, the sensing heads were washed with a $2 \mathrm{M} \mathrm{HCl}$ solution for $30 \mathrm{~min}$, to regenerate the probe for re-use and re-incubate with $10 \mathrm{nM}$ EcOMPs. The shift of the resonance was measured again to verify if the probe was able to rebind to the probe. According to the LPG principle the removal of the EcOMPs decrease the SRI and consequently the $\lambda_{R}$ shifts to upper values of wavelength, as can be seen in Fig. 5 .

As can be seen the wavelength increases with the removal of the EcOMPs and decreases again, to the same value, after the immobilization of the EcOMPs, which demonstrates the surface is entirely regenerated after $30 \mathrm{~min}$ and capable of detecting EcOMPs

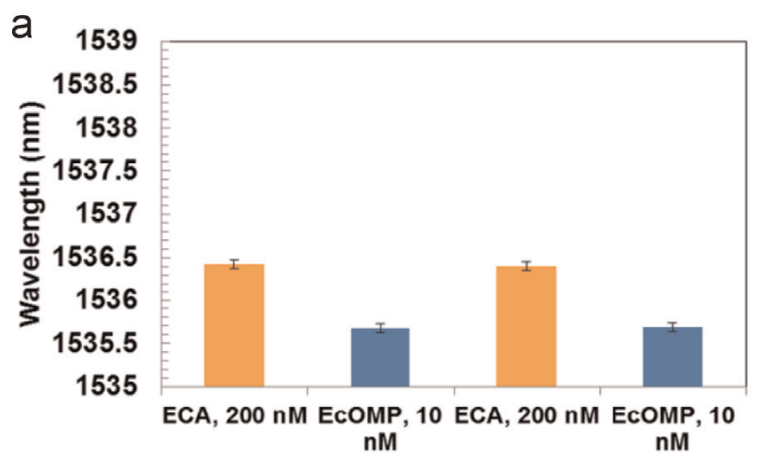

again with the same sensitivity, through at least 3 trials (consecutive and after regeneration). This assay was performed for both sensing heads and the same behavior was verified. These sensing heads shows good stability and regeneration due to their ability to detect EcOMPs in successive binding and washing events.

\subsection{Cross-reactivity to other molecules}

The aptamer herein used have been previously tested by Bruno et al., 2010, against other bacteria and has been demonstrated that this aptamer do not bind Salmonella enterica nor Campylobacter jejuni. To assess the non-specific binding of other molecules that could be present in water systems the different sensing heads were incubated with $1 \mathrm{nM}$ EcOMPs and with $1 \mathrm{nM}$ of Microcystin-Leucine, Arginine (Mcyst-LR) and with a mixture of both for $60 \mathrm{~min}$ in the affinity buffer. This Mcyst-LR concentration is the recommended maximum level of Mcyst-LR in waters established by (WHO - World Health Organization, 1998). The shift of $\lambda_{R}$ induced by the complex ECAEcOMPs for both types of sensing heads was much higher (about 95\%) than the shift obtained when incubating in Mcyst-LR solution alone. In the case of the mixture EcOMPs-Mcyst-LR the shift of the $\lambda_{R}$ presented a similar value to the complexes ECA-EcOMPs. These results show the specificity of EcOMPs binding to the DNA aptamer even in the presence of potential interferences.

\subsection{Detection of EcOMPs in environmental water samples}

In order to investigate the application of the present method to the analysis of environmental waters ( $\mathrm{pH} 6.5$ ), spiked waters were tested and the corresponding relative errors calculated. Environmental water samples were collected in a well and stored in amber glass bottles previously rinsed with double-deionized water. The $\mathrm{pH}$ and ionic strength of the water samples were adjusted by addition of affinity buffer (50:50), and they were stored at $4{ }^{\circ} \mathrm{C}$ until analysis. These assays were carried out with sensors calibrated within $0.1 \mathrm{nM}$ to $30 \mathrm{nM}$ of EcOMPs, Fig. 6a) and b).

The average recoveries (recovery is the value of the variance between the concentration measured with the sensor and the concentration of the spiked solution used to test real samples) of three spiked solutions for SH1 and SH2 were $123.39 \%$ and $106.06 \%$, respectively, thus presenting an error of about $23.39 \%$ for $\mathrm{SH} 1$ and $6.06 \%$ for SH2. A closer looks at the results, however, shows that the recovery in the lower concentration range is much better, and that the error increases as the concentration increases. This results from the fact that the assays were made with the sensors being successively exposed to higher concentrations with no prior regeneration. For this reason there is a cumulative error and some saturation effect. Therefore, in the region of higher interest, at the

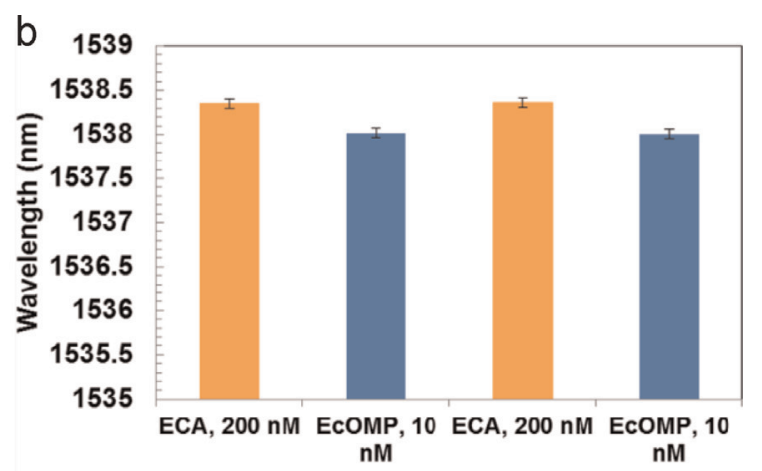

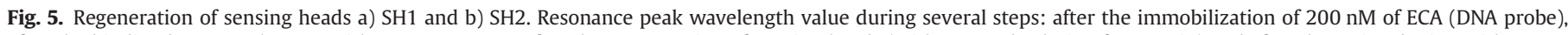

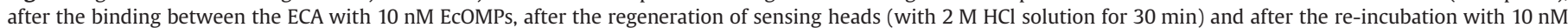
EcOMPs. The red arrow represent the moment where the HCL was added. 

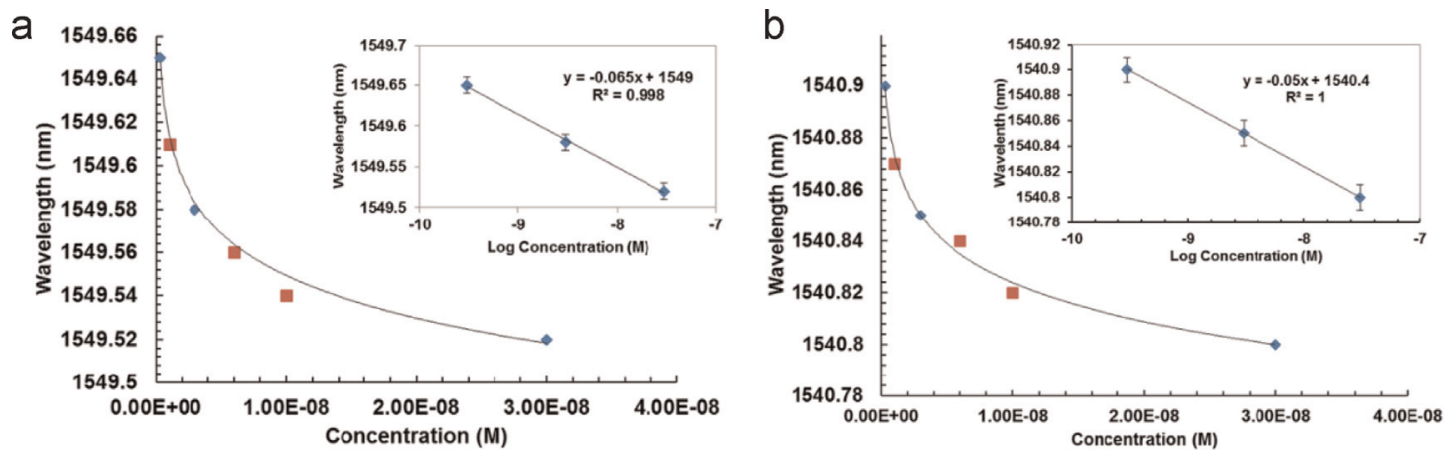

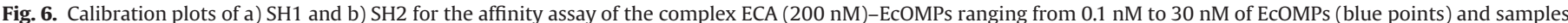

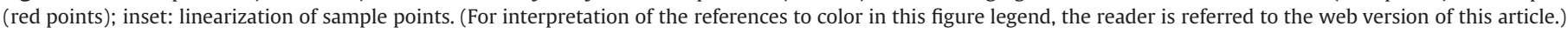

lowest concentrations the sensor performance was at its best with a very good recovery rate.

The performance and applicability of the developed biosensor was not compromise once the analysis allows the detection of $E$. coli proteins and, in the case of drinking waters, the simple presence of $E$. coli bacteria in a sample invalidate the use of this water. Therefore, in this case a presence/absence test is sufficient to analyze the sample. In addition, if the proposed use of the sensor will be the continuous monitoring of bathing waters, it is necessary to find a correlation between EcOMPs and E. coli cells, since the legislated parametric values are expressed in CFU colony forming units, to complement the analysis.

\section{Conclusions}

A label-free biosensor using a LPG as a refractometric platform for the detection of EcOMPs based on the specific recognition between a DNA aptamer capture probe and bacteria proteins was proposed. In general terms, $\mathrm{SH} 1$ presented better results in terms of reproducibility and uniformity of functionalization, but nevertheless both sensing heads have an identical performance in terms of detection. The biosensor SH2, offered more accurate results in the analysis of real samples. The sensing heads were regenerated (under low $\mathrm{pH}$ conditions) and the deviation of the subsequent detection was less than $0.1 \%$. Moreover the sensors offered simplicity in designing and analysis as well as low limit of detection. The performance and applicability of the developed biosensor allows the detection of $E$. coli proteins proving its use as a preliminary alarm system of the presence of $E$. coli in waters.

These analytical features, as well as its fabrication easiness and operational convenience, make it a promising method for the detection and quantification of $E$. coli outer membrane proteins.

\section{Acknowledgments}

R.B. Queirós thanks the Portuguese funding agency Fundação para a Ciência e a Tecnologia (FCT) for the PhD grant SFRH/BD/ $49072 / 2008$ and by the support of Project "NORTE-07-0124FEDER-000058" financed by the North Portugal Regional Operational Programme (ON.2 - O Novo Norte), under the National Strategic Reference Framework (NSRF), through the European Regional Development Fund (ERDF), FCOMP-01-0124-FEDER022701, and project Winebiocode, FCT PTDC/AGR-ALI/117341/
2010, FCOMP-01-0124-FEDER-019439. The authors also thank Professor Doctor Olga Lage for his help with the extraction of proteins from E. coli cells.

\section{Appendix A. Supplementary information}

Supplementary data associated with this article can be found in the online version at http://dx.doi.org/10.1016/j.bios.2014.06.062.

\section{References}

Baldini, F., Brenci, M., Chiavaioli, F., Giannetti, A., Trono, C., 2012. Anal. Bioanal. Chem. 402, 109-116.

Bhatia, V., Vengsarkar, A.M., 1996. Opt. Lett. 21, 692-694.

Bruno, J.G., Carrillo, M.P., Phillips, T., Andrews, C.J., 2010. J. Fluoresc. 20, 1211-1223.

Caldas, P., Jorge, P.A.S., Araújo, F.M., Ferreira, L.A., Rego, G., Santos, J.L., 2009. Meas. Sci. Technol. 20 (7), 2738-2743.

Chryssis, A.N., Saini, S.S., Lee, S.M., Yi, H.M., Bentley, W.E., Dagenais, M., 2005. IEEE J. Sel. Top. Quantum Electron. 11 (4), 864-872.

DeLisa, M.P., Zhang, Z., Shiloach, M., Pilevar, S., Davis, C.C., Sirkis, J.S., Bentley, W.E., 2000. Anal. Chem. 72 (13), 2895-2900.

El-Kashef, H., 2000. Physica B: Condens. Matter 279, 295-301.

Erdogan, T., 1997. J. Opt. Soc. Am. A 14 (8), 1760-1773.

Fan, X., White, I.M., Shopova, S.I., Zhu, H., Suter, J.D., Sun, Y., 2008. Anal. Chim. Acta $620,8-26$

Fogg, E.T., Hixson, A.N., Thompson, A.R., 1955. Anal Chem 27, 1609-1611.

Gouveia, C., 2013. Refractometric Platforms for Label-Free Biochemical Sensing (Ph. D. thesis). Centro de Ciências Exactas e da Engenharia, Universidade da Madeira, Universidade da Madeira.

Hermanson, G.T., 1996. Bioconjugate Techniquesfirst ed. Academic Press Inc., New York. Jing, M., Bowser, M.T., 2011. Anal. Chim. Acta 686, 9-18.

Mattson, G., Conklin, E., Desai, S., Nielander, G., Savage, D., Morgensen, S., 1993. Mol. Biol. Rep. 17, 167-183.

Patrick, H.J., Kersey, A.D., Bucholtz, F., 1998. J. Lightwave Technol. 16, 1606-1998.

Pilla, P., Trono, C., Baldini, F., Chiavaioli, F., Giordano, M., Cusano, A., 2012. Opt. Lett. 37, 4152-4154.

Queirós, R.B., de-los-Santos-Álvarez, N., Noronha, J.P., Sales, M.G.F., 2013. Sens. Actuators B: Chem. 181, 766-772.

Rego, G., Okhotnikov, O., Dianov, E., Sulimov, V., 2001. J Lightwave Technol. 19 (10), 1574-1579.

Shu, X., Zhang, L., Bennion, I., 2002. J. Lightwave Technol 20, 255-266.

Slavík, R., Homola, J., Ctyroky, J., Brynda, E., 2001. Sens. Actuators B: Chem. 74, 106111.

Vengsarkar, A.M., Lemaire, P.J., Judkins, J.B., Bhatia, V., Erdogan, T., Sipe., J.E., 1996. J. Lightwave Technol. 14 (1), 58-65.

White, I.M., Fan, X., 2008. Opt. Express 16 (2), 1020-1028.

WHO- World Health Organization, 1998. Drinking-Water Quality Guidelines for Parameters of Concern in Agricultural Drainage Water-Annex 3281-289 (Technical Report)

Zibaii, M.I., Kazemi, A., Latifi, H., Azar, M.K., Hosseini, S.M., Ghezelaiagh, M.H., 2010. J. Photochem. Photobiol. B: Biol. 101 (3), 313-320. 